\title{
EDITORIAL
}

\section{Special Issue on Mobile Mixed Reality}

\author{
Dr Thomas Cochrane ${ }^{a *}$, Dr Fiona Smart ${ }^{\mathrm{b}}$ and Dr Vickel Narayan ${ }^{\mathrm{a}}$ \\ ${ }^{a}$ Centre for Learning And Teaching, Auckland University of Technology; \\ ${ }^{b}$ Department of Learning and Teaching Enhancement, Edinburgh Napier University.
}

\begin{abstract}
This special collection of Research In Learning Technology explores the state of the art of mobile mixed reality (MMR) in education. The special collection includes eight articles that cover; a systematic review of MMR in healthcare higher education, using mobile devices for connecting people to places, and a variety of case studies of implementing MMR in educational contexts. The range of papers illustrates the emergence of MMR as a platform for designing authentic learning environments in both formal and informal learning situations. The papers also highlight a general lack of engagement with new learning theories and models in the use of MMR to design transformative learning experiences.
\end{abstract}

Keywords: design-based research; scholarship of technology enhanced learning; augmented reality; virtual reality; mixed reality; mobile learning

\section{Introduction}

Mobile mixed reality is part of what has become popularly termed immersive reality (Peña-Rios et al., 2016), encompassing a continuum from augmented reality to virtual reality. MMR is part of long established education strategies that include simulation as an established educational methodology utilised particularly in healthcare higher education (Kaufman, 2010, Laschinger et al., 2008). The three main limitations of simulation - authenticity, access, and cost can be mitigated by utilising the affordances of MMR enabled by the development of mobile devices into powerful multi-sensory ubiquitously connected computing devices. In particular, MMR provides a potential practical and cost-effective solution to design innovative and authentic training environments (Plahouras et al., 2018, Barr and Foster, 2017, Birt et al., 2017). Augmented and virtual reality (mixed reality) is an emerging technology that bridges the gap between computer generated and real-world environments (Uluyol \& Sahin, 2016). Mobile Mixed Reality enables the design of authentic learning environments that explore the impact of socio-culture influences, and lead to deeper student engagement with the real world via digitally enhanced gamified environments. This is illustrated by the phenomenon of Pokemon Go, and emergent mobile mixed reality projects with the likes of the Microsoft Hololens and Google Cardboard. The use of AR and VR to support teaching and learning has shown to have many advantages which include enhancement of learning achievement in terms of enhanced learning outcomes, motivation and engagement (Bacca, Baldiris, Fabregat, Graf, \& Kinshuk, 2014). 


\section{Dr Thomas Cochrane et al.}

Interest in the application of VR and AR have increased dramatically over the last few years (Akçayır \& Akçayır, 2017). However due to the relatively emerging nature of these technologies the implications of these tools in education are still largely still being explored. The RLT special collection on MMR builds upon the publication (November 2017) of a special issue of AJET guest edited by Dr Thomas Cochrane and Dr Helen Farley, with the theme: "Exploring Mobile Augmented and Virtual Reality in Higher Education" (Cochrane and Farley, 2017). In this special collection we explore the design of educational experiences that integrate these MMR technologies into both formal and informal learning environments.

\section{Mobile Mixed Reality in Education}

AR and VR are currently two of the hottest topics in emerging mobile learning technologies. Coupled with the ubiquity of mobile device ownership, mobile AR and VR provide the potential to enhance educational technology research and practice on a level previously impractical. By focusing upon design-based research the special issue focuses on new emancipative pedagogies beyond adding another technology fad for content delivery and teacher-directed pedagogies. Design based research facilitates the design of authentic innovation in teaching and learning rather than research that is technology driven or merely substitutionary (Rushby, 2012, Traxler, 2016, Bannan et al., 2015).

Mobile devices have been considered as especially well-suited to engaging with virtual and augmented environments due to their accessibility, portability and their ability to immerse students into their own personal environment but still engage with others (Furió, González-Gancedo, Juan, Seguí, \& Costa, 2013). Due to the pervasive adoption of mobile technology the focus on mobile devices and a BYOD (Bring Your Own Device) student-owned model ensures inclusivity (Traxler, 2010, 2016a). Many of the emergent mobile AR and VR projects such as Google Expeditions include the potential for international collaborations and partnerships. For example, mobile mixed reality enables students to experience authentic scenarios, field trips, and simulations from across the globe. This special collection explores some of the emergent examples of the design of MMR learning environments.

\section{In This Issue}

Here we explore the emergent themes drawn from the eight articles included in this special collection.

\section{Triangulating the impact of MMR learning through biometric feedback}

In their paper "Embodied reports in Paramedicine Mixed Reality learning" Aguayo et al., (2018) describe a pilot project that is part of a design based research project exploring the impact of mobile VR simulation learning environments in Paramedicine education utilizing both self-reported and biometric feedback data. They highlight the shift in thinking behind the project through the DBR iterations from a focus upon VR to a focus upon mixed reality (MR) through the addition of biometric feedback that anchors the project in both the virtual and real spaces. They argue for the 
value of incorporating biometric feedback to enhance the design of authentic learning experiences through mobile mixed reality.

\section{Evaluating learning in virtual reality}

In their paper "Learning in Virtual Reality: Effects on Performance, Emotion, and Engagement", Allcoat and von Muhlenen (2018) compared the impact of three different modes of learning for students, including: traditional textbook-style, VR, and video. They found that overall, participants found VR learning an improved learning experience compared to traditional textbook and video learning methods, resulting in longer-term benefits for learners. However, they caution that the results may be partially due to the novelty factor of VR, and longitudinal studies are required to explore this further.

\section{Assessing mobile mixed reality affordances}

In their paper "Assessing mobile mixed reality affordances as a comparative visualization pedagogy for design communication", Birt and Cowling (2018) use a design based research methodology to explore mixed reality approaches to enhancing design student spatial recognition skills. They evaluate the impact and use of a range of mobile comparative visualization technologies within a first-year architectural design classroom. Their findings highlight the benefit of MR visualisation for exposing layers of complex architectural designs, and how these visualisations push the limits of current mobile technology in comparison to dedicated non-mobile devices. Future research plans are to explore the potential of designing collaboration within these MR learning environments.

\section{Designing authentic interdisciplinary health education scenarios}

In their paper "Authentic Interdisciplinary Health Education Scenarios using Mobile VR", Cochrane et al., (2018) explore the potential of mobile mixed reality environments for designing authentic interdisciplinary team interaction that mirror professional practice but are difficult to achieve in the typically dislocated university department structure where each health discipline is separated. They present a design based research methodology that leverages mobile social media to produce an agile, cost-effective and scalable model to design MMR learning environments for transdisciplinary team collaboration.

\section{Trialing the Hololens}

In their paper "Holographic Learning: A Mixed Reality Trial of Microsoft HoloLens in an Australian Secondary School", Leonard and Fitzgerald (2018) evaluate the potential of the HoloLens in education through a pilot phase of a design based research project. They found the main limitation of AR and VR technologies in secondary education is the dominant default use of the technology to design for teacherdirected pedagogy or Isocratic learning. The authors argue that their observations of the pilot project indicate a general lack of engagement of teachers with diverse 


\section{Dr Thomas Cochrane et al.}

models for designing learning experiences and environments that allow for student customisation and uncertainty in the learning experience. In response the authors appeal for teachers to utilise new technologies to design transformative learning experiences, moving beyond substitutionary use of new technologies for existing pedagogical practice.

\section{Using mobile devices for connecting people to places}

In their paper "The Boundaries of Education: Using mobile devices for connecting people to places", Sinfield (2018) explores the use of MMR technologies to mediate learning outside of formal classroom environments through a sociocultural lens that links a community to its history. The paper outlines a potentially transferable framework for using MMR to design authentic informal learning environments. In this project MMR is used to capture and bring to life significant aspects of a communities past, linking the past to the present in the design of a highly interactive learning environment. The author argues that MMR technologies can be used by sociocultural researchers in a variety of contexts and informal learning environments.

\section{A systematic review of MMR in health education}

In their paper "Exploring Mobile Mixed Reality in Healthcare Higher Education: A Systematic Review", Stretton et al., (2018) describe the results of a systematic review of the literature surrounding mobile learning and mixed reality in healthcare higher education. From an initial 1484 studies 18 were identified that met the inclusion criteria focused on "healthcare", "higher education", "mobile learning" and "mixed reality". Their critique of these studies indicates that MMR is predominantly utilised in healthcare education for clinical simulation exercises, with a focus upon skill development rather than higher level diagnosis or higher order clinical reasoning. Consequently, the authors identify that there is little evidence of engagement with learning theory in the design of MMR for HHE, and a subsequent reliance upon behavioural learning design.

\section{Immersive mobile displays}

In their paper "Using immersive mobile displays for observational learning of motor skills. A design research approach enhancing memory recall and usability", Watson and Livingston (2018) explore the effectiveness of MMR platforms to facilitate observational learning of action sequences. The paper evaluates the development of the Recovery Position Application that displays two virtual avatars performing the steps of the recovery position as an example of MMR technologies used for virtual demonstrations. Based upon their research, the authors develop guidelines for virtual demonstration to supplement instructor demonstrations beyond simple recorded videos of demonstrations. The authors argue that $3 \mathrm{D}$ virtual environments can provide effective learning environments for specific training experiences. 


\section{Acknowledgements}

The special issue guest editors would like to express our thanks to the RLT editorial team for allowing us to explore a special issue on MMR. Special thanks also go to our team of reviewers and authors for getting the special issue through in such a short time-frame.

\section{References}

Aguayo, C., Dañobeitia, C., Cochrane, T., Aiello, S., Cook, S. \& Cuevas, A. 2018. Embodied reports in paramedicine mixed reality learning. Research in Learning Technology, 26.

Allcoat, D. \& Mühlenen, A. V. 2018. Learning in Virtual Reality: Effects on Performance, Emotion, and Engagement. Research in Learning Technology, 26.

Bannan, B., Cook, J. \& Pachler, N. 2015. Reconceptualizing design research in the age of mobile learning. Interactive Learning Environments, 24, 1-16.

Barr, N. \& Foster, J.-A. 2017. Using consensus group methods to improve simulation using immersive media in paramedicine. Journal of Paramedic Practice, 9, 121-125.

Birt, J. \& Cowling, M. 2018. Assessing mobile mixed reality affordances as a comparative visualization pedagogy for design communication. Research in Learning Technology, 26.

Birt, J., Moore, E. \& Cowling, M. 2017. Improving paramedic distance education through mobile mixed reality simulation. Australasian Journal of Educational Technology (AJET), 33, 69-83.

Cochrane, T. \& Farley, H. 2017. Editorial: Volume 33 Special Issue on Mobile AR \& VR Integrating SOTEL in learning design. Australasian Journal of Educational Technology (AJET), 33.

Cochrane, T., Stretton, T., Aiello, S., Britnell, S., Cook, S. \& Narayan, V. 2018. Authentic Interprofessional Health Education Scenarios using Mobile VR. Research in Learning Technology, 26.

Kaufman, D. 2010. Simulation in Health Professional Education. In: Kaufman, D. \& Sauvé, L. (eds.) Educational Gameplay and Simulation Environments: Case Studies and Lessons Learned. Hershey, PA, USA: IGI Global.

Laschinger, S., Medves, J., Pulling, C., Mcgraw, D. R., Waytuck, B., Harrison, M. B. \& Gambeta, K. 2008. Effectiveness of simulation on health profession students' knowledge, skills, confidence and satisfaction. International Journal of Evidence-Based Healthcare, 6, 278-302.

Leonard, S. N. \& Fitzgerald, R. N. 2018. Holographic Learning: A Mixed Reality Trial of Microsoft HoloLens in an Australian Secondary School. Research in Learning Technology, 26.

Peña-Rios, A., Pirker, J., Gütl, C. \& Gardner, M. 2016. Editorial: Preface to the Special Issue on Immersive Environments: Challenges, Research and New Developments. EAI Endorsed Transactions on Future Intelligent Educational Environments, 2, 1-2.

Plahouras, J., Khan, R., Johnston, B. C., Scaffidi, M. A., Grover, S. C. \& Walsh, C. M. 2018. PTH-140 Virtual reality simulation training in gastrointestinal endoscopy: a cochrane systematic review. Gut, 67, A275.

Rushby, N. 2012. Editorial: An agenda for mobile learning. British Journal of Educational Technology, 43, 355-356.

Sinfield, D. 2018. The Boundaries of Education: Using mobile devices for connecting people to places. Research in Learning Technology, 26.

Stretton, T., Cochrane, T. \& Narayan, V. 2018. Exploring Mobile Mixed Reality in Healthcare Higher Education: A Systematic Review. Research in Learning Technology, 26.

Traxler, J. 2016. What killed the mobile learning dream? [Online]. Available: https://www.jisc. ac.uk/inform-feature/what-killed-the-mobile-learning-dream-26-feb-2016?

Watson, P. \& Livingstone, D. 2018. Using mixed reality displays for observational learning of motor skills: A design research approach enhancing memory recall and usability. Research in Learning Technology, 26. 